\title{
Menschen für ein gutes Ergehen veranlagen
}

\section{Ein Kommentar zu Wohlstandsberichten}

WOLF RAINER WENDT

Prof. Dr. Wolf Rainer Wendt ist Vorsitzender der Deutschen Gesellschaft für Care und Case Management sowie Honorarprofessor der Eberhard Karls Universität Tübingen. Er ist Mitbegründer und langjähriger Vorsitzender der Deutschen Gesellschaft für Soziale Arbeit. Seit über drei Jahrzehnten ist er Mitglied im Beirat der Blätter der Wohlfahrtspflege.

E-Mail prof.dr.wendt@googlemail.com

\author{
Früherziehung besteht wesentlich in einer \\ förderlichen Gestaltung der kindlichen \\ Umgebung, bestimmt von den Eltern und von den \\ frühpädagogischen Institutionen. Deren Arbeit \\ setzt nicht zuletzt die Eltern in den Stand, ihrerseits \\ zum Wohl des Kindes besser in der Erziehung \\ zurechtzukommen. Diese Aspekte werden im \\ ökonomischen Diskurs leicht übersehen.
}

Im ökonomischen Diskurs bemüht man sich seit einigen Jahren, in der Volkswirtschaftlichen Gesamtrechnung vom Bruttoinlandsprodukt (BIP) weg zu einer besseren Erfassung von Wohlstand in der Gesellschaft und Wohlfahrt der Menschen zu gelangen.

Schritte wurden mit dem Human Development Index, der Verwirklichungschancen erfassen soll, und von der Stiglitz/Sen/Fitoussi-Kommission (2010) unternommen. Am Diskurs beteiligt war von 2010 bis 2013 die EnqueteKommission »Wachstum, Wohlstand, Lebensqualität« des Deutschen Bundestages; es gibt deutsche und britische Studien, nationales Wohlergehen zu messen (Diefenbacher/Ziehschank 2011, Harper/Price 2011, ONS 2011) und des weiteren eine »Beyond GDP«-Initiative der Europäischen Kommission.

Seit Mitte 2012 liegt der sehr ausführliche Inclusive Wealth Report des UN University International Human Dimensions Programme on Global Environmental Change (UNU-IHOP) und des UN Environment Programme (UNEP) vor. Die Darlegungen in diesem »Gesamtvermögensbericht " wie in den anderen Studien sind bedeutsam für das Verständnis sozialer Leistungserbringung und der Produktivität von organisierter Wohlfahrtspflege in ihrem Beitrag für ein gutes Ergehen von Menschen.
Der »Reichtum der Nationen", den einst Adam Smith in marktlich vermittelter arbeitsteiliger Produktion begründet sah, zehrt von Ressourcen, die begrenzt sind und gepflegt sein wollen. Worauf kann eine Ökonomie in der Erzeugung von Wohlstand zurückgreifen und bauen?

\section{Soziale Aktivposten im gesamtwirtschaftlichen Vermögen}

Der Inclusive Wealth Report (IWR) erfasst die Aktivposten für eine nachhaltige Wohlstandsentwicklung in drei Dimensionen und Formen von Kapital: Das Vermögen liege in den erstellten Produkten, in den Menschen und in der Natur vorhandenen Kapitals. Die gleiche Einteilung nehmen die britischen Studien vor (Harper/Price 2011, S. 6). »Wealth « als Reichtum oder Wohlstand wird im Inclusive Wealth Report als gesellschaftlicher Wert dieser ökonomischen Wertbestände bestimmt. $\mathrm{Zu}$ ihnen gerechnet werden sieben Aktivposten: »das reproduzierbare Kapital, Humankapital, Wissen, Naturkapital, Bevölkerung, Institutionen und Zeit" (IWR, S.XXIII). Die Studie nennt sie die "produktive Basis « einer Nation und ihrer Ökonomie. Der Bericht bewegt sich auf der Aggregatebene von Nationen, wenngleich er dabei das Wohlergehen der Bürgerinnen und Bürger einzeln und gemeinsam im Blick haben will. $\rightarrow$ 
Auf die Beziehung von Wohlergehen auf Wohlstand (»well-being and wealth«) geht das von P. Dasgupta und A. Duraiappah verfasste erste Kapitel des Inclusive Wealth Report ein. Die Aktivposten des Wohlstandes fundieren die ökonomischen (erwerbswirtschaftlichen) Produktionsprozesse, deren Früchte unter dem Einfluss kultureller und anderer institutioneller Normen zum Wohlergehen der Bevölkerung beitragen. Das Kapitel beschreibt die
Arten als Mittel zu begreifen sind, mit denen diese Güter und Dienste erstellt werden (IWR, S. 18). Per Konsum werden sie genutzt (und was sie dabei jeweils zum Wohlergehen beitragen, wird in einem "Schattenpreis « ermessen).

Der UN-Bericht hält sich nicht bei der "widersprüchlichen Vorstellung von Bedürfnissen " auf, denen das wirtschaftliche Handeln nachkommt, sondern bestimmt den Konsum im weiten Sinne der Nutzung nicht allein der ma-

\section{"Lebensqualität und Humanvermögen sind die beiden Hauptkomponenten von Wertschöpfung in der Wohlfahrtspflege"}

sieben Arten »Kapital « genauer und bemerkt zum »human capital (education, skills, tacit knowledge, health) «, es sei in den Menschen verkörpert und könne, wie jedem Lehrer schmerzlich bewusst sei, nur mit Kosten von einer Person auf eine andere übertragen werden. Bildung, Befähigungen und Gesundheit seien zugleich Ziele und Mittel, die Ziele zu erreichen. Einerseits von intrinsischem Wert lohne ihr Einsatz, weil damit die Produktivität einer Person erhöht werde (IWR, S.16). Investitionen beispielsweise in Früherziehung dienen danach letztlich dem ökonomischen Wachstum.

Die Schwierigkeit dieser Argumentation tritt anschließend bei Betrachtung des Aktivpostens Bevölkerung (Größe und demografisches Profil) hervor: »Konzeptuell bereitet diese Kategorie die größten Probleme, weil die Menschen einerseits der Grund sind, Wohlergehen ins Zentrum der Nachhaltigkeitsanalyse zu rücken, und andererseits Mittel sind zur Realisierung von Wohlergehen.« Das Arbeitskräftepotenzial eines Landes erscheint als Bedingung des Wohles der Bevölkerung. Indes lassen sich individuelle und soziale Wohlfahrt und wirtschaftliche Produktivität, wie sie herkömmlich (und in der ökonomischen Wohlfahrtstheorie) verstanden wird, so leicht nicht abgleichen.

\section{Wohlergehen per Konsum}

Menschliches Wohlergehen wird in der Studie bestimmt als "a function of the consumption and use of goods and services«, wonach die genannten Kapital- teriellen Güter, sondern auch von Freizeit, sozialen Beziehungen, geschützter Umwelt u. a. m. (IWR, S. 6) als Entfaltungsmöglichkeit individueller und gemeinsamer Wohlfahrt.

Der Inclusive Wealth Report argumentiert volkswirtschaftlich und somit auf der Aggregatebene. Er behandelt, was in einer Nation deren nachhaltiger Entwicklung förderlich ist und den Menschen insgesamt geboten wird. Insoweit erscheinen sie alle gewissermaßen als "Wohlfahrtsempfänger«. Außer Betracht bleibt der produktive Prozess, in dem Menschen selber sich um ihr Wohl kümmern, ihr Ergehen in eigener Lebensführung bestimmen und für sich, miteinander und füreinander sorgen. Kein Thema ist die Generierung und Produktivität des Sozialen selber.

Unter Sozialkapital werden im Inclusive Wealth Report Aspekte sozialer Strukturen verstanden, die dem ökonomischen Handeln förderlich sind. Die individuellen Vermögensbestände (an Bildung, Befähigungen, Gesundheit usw.) haben zwar erwerbswirtschaftlich - per Angebot auf dem Arbeitsmarkt - Gewicht, aber das sie unterhaltende und pflegende Geschehen in Personenhaushalten tritt marktvermittelt nicht in Erscheinung. Was in sozialer und persönlicher Lebenspraxis an Wohlfahrt zustande kommt, interessiert diesseits der Bereitstellungsund Lieferbeziehungen marktlichen Wirtschaftens nicht. Demgegenüber ist anderweitig ausgeführt worden, dass im sorgenden Handeln auf der Mik- roebene menschlicher Beziehungen der »wahre Reichtum der Nationen " (Eisler 2008) besteht.

Der Inclusive Wealth Report behandelt die Vermögen zu nachhaltigem Wirtschaften. Wie man mit einer Nachhaltigkeits-Strategie vorankommt, wird in den drei Dimensionen Ökonomie, Ökologie und Soziales erfasst. Analog hält sich die Enquete-Kommission des Bundestages bei der Entwicklung eines ganzheitlichen »Wohlstands- und Fortschrittsindikators" an die Bereiche "materieller Wohlstand « (mit den Leitindikatoren Bruttoinlandsprodukt, Einkommensverteilung und Staatsschulden), "Soziales und Teilhabe « (mit den Leitindikatoren Beschäftigungsquote, Bildungsniveau, Gesundheit, Freiheit) und "Ökologie" (mit den Variablen Treibhausgase, Stickstoff und Artenvielfalt).

Hier wie dort wird makroökonomisch auf Bestands- und Stromgrößen gesehen und unterstellt, dass in dem mit ihnen gekennzeichneten Betrieb die Menschen (mehr oder weniger) "gut leben«.

\section{Chancen, gut zurechtzukommen - Beispiel Früherziehung}

Es handelt sich bei all dem zweifellos um Faktoren, die für das individuelle Wohlergehen bedeutsam sind. Aber der einzelne Mensch ist in sehr unterschiedlichem Maße in der Lage, das in seinem Umfeld und bei ihm vorhandenes »Vermögen« zu realisieren. Personen nutzen es nicht einfach, sondern sind selber »Vermögensschöpfer « im Interesse eigener Wohlfahrt.

Persönliche Vermögensbestände an Bildung, Befähigung und Gesundheit entwickeln sich in der aktiven Wechselbeziehung von Individuum und seiner relevanten Umwelt. Der personenbezogene Prozess der Entwicklung und des Unterhalts von Wohlfahrt wird übersehen, wenn nur das Kapital insgesamt betrachtet wird, das wirtschaftlich verfügbar ist und in der Gesellschaft für Wohlfahrt eingesetzt werden kann. Das ist die oben genannte Krux mit dem Aktivposten »Bevölkerung «: Seine erwerbswirtschaftliche Nutzung und die des »Humankapitals « erbringt nur mittelbar und in sehr unterschiedlichem Maße eine Wohlfahrt für den Einzelnen mit sich. Er erstellt sie selber in seiner Lebensführung, soweit es ihm die Umstände erlauben oder wie sie ihn förderlich dahin bringen. 
Nehmen wir das Beispiel der Früherziehung. Sie besteht wesentlich in einer förderlichen Gestaltung der kindlichen Umgebung, bestimmt von den Eltern und komplementär von frühpädagogischen Institutionen. Deren Soziale Arbeit setzt nicht zuletzt die Eltern »in den Stand «, ihrerseits zum Wohl des Kindes besser in der Erziehung zurechtzukommen. Andere Aktivposten wie der Erwerbsstatus der Familie, Gesundheit, Wissen und verfügbare Zeit sind gewichtige Variable der kindlichen Verwirklichungschancen. Eine veranstaltete Früherziehung sorgt somit nicht durch sich allein für das Wohlergehen ihrer Adressaten, sondern erfolgversprechend nur in einer Interaktion, etwa einer Bildungspartnerschaft, mit dem Elternhaus, das seinerseits in soziale Beziehungen und Aktivitäten eingebunden ist.

In dieser Komplexität ist der soziale Prozess ein produktiver: Er erzeugt Lebensqualität und Humanvermögen, die beiden Hauptkomponenten von Wertschöpfung in der Wohlfahrtspflege.

\section{Soziale Produktivität diesseits der Stromgrößen des Kapitals}

Soziale Wechselbeziehungen bilden sich im marktvermittelten Wirtschaftskreislauf nicht ab. Wenn Familien und Sozialprofessionelle sich um Kinder kümmern, lassen sich von diesem Geschehen zwar der Input und Output des Kapitals (in Form der Vermögensbestände im Inclusive Wealth Report) messen. Eine Be- wertung, wie es den Kindern in diesem Geschehen ergeht, ihre Wohlfahrt, wird so nicht erfasst. Sie ist nicht lieferbar und konsumierbar.

Am Beispiel des Aktivpostens Bildung wird der Unterschied deutlich, der zwischen einem messbaren Bildungsstand in der Bevölkerung sowie dem Niveau von Bildungseinrichtungen einerseits und der persönlichen Bildung von Menschen als Befähigung und Verhaltensmerkmal andererseits besteht. In individueller Wohlfahrt bündelt Bildung die Verwirklichungschancen der Lebensgestaltung. Zustande kommen sie in einem eigenständigen Prozess auf der Individualebene, den die Stromgröße des Bildungskapitals auf der Makroebene nicht abbilden kann.

Analog bei anderen Tatbeständen des Sozialkapitals: Betreuung, Pflege, sozialer Austausch und die Realisierung von Teilhabe tragen wesentlich zum Unterhalt von Wohlfahrt bei (vgl. Wendt 2011). Die Bereitstellung von personellen und sächlichen Ressourcen dafür und der Aufwand, der insgesamt getrieben wird, ermisst nicht das Wohlbefinden und die Qualität des Lebens derjenigen, die entsprechende Leistungen empfangen.

Es ist die Eigenart des Sozialen, dass es sich selber fortzeugt und die an ihm Beteiligten unterhält. Die Kapitalbestände und insgesamt der Reichtum, den die Wohlstandsberichte makroökonomisch erfassen, lassen sich nutzen - u. a. auf sozialem Gebiet. Wie

\section{Elemente der Sozialwirtschaft}

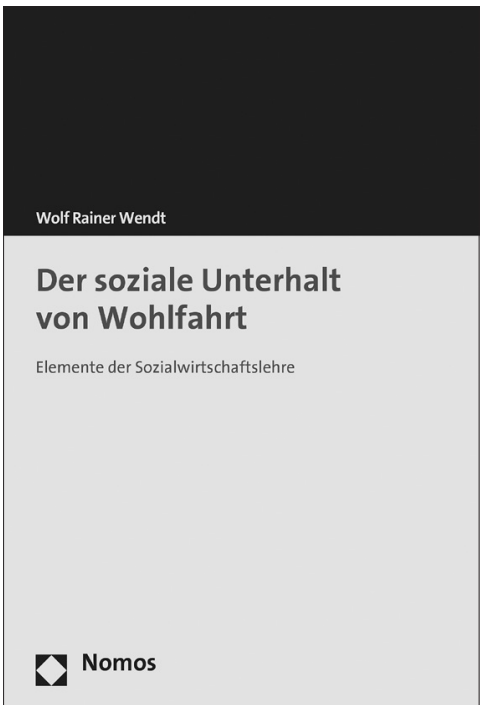

Sozialwirtschaft umfasst im System der sozialen Sicherung und Versorgung die Vielfalt der Unternehmungen und Leistungen zur individuellen und gemeinschaftliche Wohlfahrt. Für ihren Unterhalt stehen im Sozialund Gesundheitswesen die Organisationen, Einrichtungen und Dienste der öffentlichen und sozialen Daseinsvorsorge ein. Die Pflege von Wohlfahrt in "Diensten am Menschen" ist produktiv. Ihre Ökonomie im Haushalt des Sozialstaats und in Beziehung auf die Lebensführung im Haushalt von Personen und in Gemeinschaft ist Gegenstand dieses Buches von Wolf Rainer Wendt.

Wolf Rainer Wendt: Der soziale Unterhalt von Wohlfahrt. Elemente der Sozialwirtschaftslehre. Nomos Verlagsgesellschaft Baden-Baden 2011. 178 Seiten. 39,- Euro. ISBN 978-3-8329-7000-0.

aber, ist eine Frage der sozialen Kultur: des Umgangs unter Menschen, persönlichen Sorgens und der Gestaltung formeller Versorgung. Hier zählt nicht die erwerbswirtschaftliche Produktivität, sondern die Wirtlichkeit von Lebensverhältnissen, in denen Menschen für ihr Wohlergehen mehr oder weniger gut veranlagt sind oder dazu veranlagt werden.

\section{Ausblick: Hoffnung auf 2014}

Wie sich das Wohlergehen von Menschen direkt fördern und ihre Versorgung nachhaltig auskömmlich gestalten lässt, ist ein Thema, das der Inclusive Wealth Report 2012 in seiner Konzentration auf Naturkapital nicht behandelt. Es kann eine Perspektive für den nächsten Bericht mit Fokus auf das "soziale Kapital « sein, den uns das UN-Programm für 2014 in Aussicht stellt.

\section{Literatur}

Diefenbacher, H./Ziehschank, R. (2011): Woran sich Wohlstand wirklich messen lässt. Alternativen zum Bruttoinlandsprodukt. München. Eisler, R. (2008): The Real Wealth of Nations. Creating a Caring Economics. New York.

Harper, G. /Price, R. (2011): A framework for understanding the social impacts of policy and their effects on wellbeing. London.

ONS (2011): Developing a Framework for Understanding and Measuring National Well-being. London.

Stiglitz, J.E./Sen, A./Fitoussi, J.-P. (2010): Mismeasuring Our Lives. Why GDP Doesn't Add Up. New York.

UNU-IHDP and UNEP: Inclusive Wealth Report 2012. Measuring progress toward sustainability. New York.

Wendt, W. R. (2011): Der soziale Unterhalt von Wohlfahrt. Baden-Baden. 